\title{
Characterizing the molecular and morphophysiological diversity of Italian red clover
}

\author{
Mario A. Pagnotta $\cdot$ Paolo Annicchiarico • \\ Anna Farina $\cdot$ Sandro Proietti
}

Received: 11 June 2010/Accepted: 20 December 2010/Published online: 29 December 2010

(C) Springer Science+Business Media B.V. 2010

\begin{abstract}
Red clover (Trifolium pratense L.) is the third major forage species in Europe, but there is limited information on the biodiversity and the genetic structure of landraces and natural populations which evolved in this region. The objective of this study was producing such information for Italian germplasm on the ground of molecular and morphophysiological diversity. The study included 16 Italian natural populations from a wide range of environments, four landraces representing the four traditional commercial ecotypes, and two varieties. Eight morphophysiological traits were assessed in a replicated trial under field conditions, whereas two AFLPs primer combinations with 140 polymorphic markers were recorded on a random sample of 13 genotypes per population. Ordination and classification results based on morphophysiological data clearly kept track of the type of germplasm (i.e. landrace or natural population) and its geographic origin, unlike results based on molecular markers. Euclidean distances among populations based on morphophysiological traits were not correlated with Nei's genetic distances
\end{abstract}

M. A. Pagnotta $(\bowtie) \cdot$ A. Farina

Department of Agrobiology and Agrochemistry,

University of Tuscia, Via San Camillo de Lellis, 01100 Viterbo, Italy

e-mail:pagnotta@unitus.it

P. Annicchiarico $\cdot$ S. Proietti

CRA-Centro di Ricerca per le Produzioni Foraggere

e Lattiero-Casearie, Viale Piacenza 29, 26900 Lodi, Italy based on molecular markers according to Mantel's test. Geographical distances among landrace or natural population material was correlated with distances based on morphophysiological traits but not with those based on molecular markers. The average within-population variation estimated via molecular markers was about 2.6-fold higher than that among populations, preventing an acceptable discrimination among most populations. On average, natural populations tended to have within-population variation similar to varieties and somewhat lower than landraces. Our results have implications for collection, conservation, exploitation and registration in a sui generis system of red clover genetic resources.

Keywords Genetic distance - Genetic resources · Genetic structure · Gene flow

\section{Introduction}

Red clover (Trifolium pratense L.) is a temperate forage species which occurs in the wild in Europe and a portion of Near East (Merkenschlager 1964). Its domestication and introduction into European farming systems dates back to the sixteenth century and first concerned Spain, northern Italy, and some regions of northwest Europe (Mousset-Déclas 1995). Red clover is a cross-pollinated, diploid species $(2 n=14)$ with a 